\title{
Effect of congenital blepharoptosis on corneal biomechanical properties and changes after ptosis surgery
}

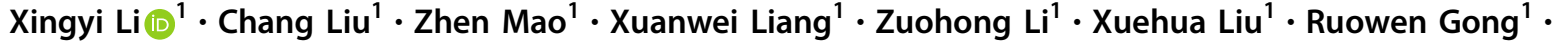 \\ Danping Huang $\mathbb{1}^{1}$
}

Received: 21 November 2018 / Revised: 11 July 2019 / Accepted: 27 August 2019 / Published online: 26 September 2019

(c) The Author(s), under exclusive licence to The Royal College of Ophthalmologists 2019

\begin{abstract}
Objectives We studied the difference in the corneal biomechanical parameters of ptotic and fellow eyes in patients with congenital blepharoptosis. The correlations between corneal biomechanical parameters and demographic or ocular parameters, and the changes after surgery were also researched.

Methods The corneal biomechanical parameters were measured by Corvis ST tonometry. The central corneal thickness (CCT), axial length (AL) and keratometry measurements were performed with LenStar LS900, and intraocular pressure (IOP) by non-contact applanation tonometry. The parameters were evaluated for the effect of ptosis and the relationship of corneal biomechanical parameters. These examinations were repeated 6 months after blepharoptosis surgery.

Results Twenty-nine patients were enroled. The Corvis ST parameters (Deformation amplitude [DA], A1 times, and A1 velocity), CCT, IOP with NCT, IOP with corrected, differed significantly between ptotic and fellow eyes. CCT was significantly positively correlated with Length A1 and IOP with Corvis, and negatively correlated with IOP corrected by Corvis of the ptotic eyes. The same tendency was found in the fellow eyes. Six months after the ptosis surgery, the differences in corneal biomechanics parameters between ptotic eyes and fellow eyes were not significantly changed.

Conclusions Congenital blepharoptosis causes significant corneal biomechanical changes measured by Corvis ST. The ptotic eyes had thicker and less deformable corneas. The differences in corneal biomechanics between ptotic eyes and fellow eyes were mostly related to CCT changes. Six months after surgery, these differences in corneal biomechanics did not change significantly.
\end{abstract}

\section{Introduction}

Congenital blepharoptosis is an eyelid disorder caused by dysgenesis of the levator muscle of the upper eyelid [1]. This condition is frequently associated with amblyopia, refractive error, anisometropia, and strabismus. Some studies have found that ptotic eyes have more severe astigmatism and more oblique astigmatism than their fellow eyes $[2,3]$. The significant difference for astigmatism at horizontal meridian between ptotic and fellow eyes is probably a consequence of gravitational pressure [4]. Amblyogenic astigmatism is common in ptotic eyes [5]. A significant

Danping Huang

hdanp@mail.sysu.edu.cn

1 State Key Laboratory of Ophthalmology, Zhongshan Ophthalmic Center, Sun Yat-Sen University, 510060 Guangzhou, China change in astigmatism has been detected after surgery, which can be a possible cause of amblyogenic astigmatism [6]. Studies of keratoconus and astigmatic corneas found that more parameters showed significant differences in an against-the-rule astigmatism group than in a normal control group [7]. Recent research has concentrated on the refraction abnormality of ptotic eyes, so the effects on corneal biomechanical properties remain uncertain.

Corvis ST tonometry (Oculus, Wetzlar, Germany) is a recently developed automated non-contact air-puff tonometer associated with ultra-high-speed Scheimpflug technology. In addition to taking IOP measurements, this tonometer provides optical pachymetry readings and in vivo information about corneal biomechanical properties. The aim of the present study was to measure the corneal biomechanical properties of congenital blepharoptosis patients using a Corvis ST. Comparing the corneal biomechanical properties of ptotic eyes and fellow eyes and follow-up after blepharoptosis surgery will allow further delineation of the 
effect of gravitational pressure of the ptotic eyelid, the relationship between ptosis severity, and corneal biomechanical properties.

\section{Subjects and methods}

The congenital blepharoptosis patients who were admitted to the oculoplastic department in Zhongshan Ophthalmic Center, Sun Yat-sen University were enroled in this prospective observational cohort study between July and August 2017. All participants provided signed informed consent. The study followed the tenets of the Declaration of Helsinki and was approved by the ethics committee of the Zhongshan Ophthalmic Center.

Full ophthalmic examinations consisted of best-corrected visual acuity measurements, slit-lamp examination, detailed fundus examination, cover test, and extraocular movements. The height of palpebral fissure (HPF), upper eyelid marginal reflex distance (MRD 1), and levator function (LF) were also evaluated twice by one senior doctor (DH). MRD 1 was the distance from the central corneal light reflex to the upper eyelid margin in the primary position of gaze in millimetres as measured in primary gaze with the frontalis muscle fixed. Blepharoptosis was defined as the presentation of an MRD 1 of $<2 \mathrm{~mm}$ as suggested by previous studies and/or an asymmetry between both upper eyelid heights of $\geq 1 \mathrm{~mm}$ in unilateral cases [8]. Congenital blepharoptosis was diagnosed and verified by a history of eyelid droop presenting at birth or from a photograph taken in the first few months of life.

The congenital blepharoptosis inclusion criteria were the following: diagnosis of congenital blepharoptosis, absence of other ocular pathology, and reliable measurement results. The exclusion criteria included a history of eye trauma, previous ocular surgery (including ptosis correction surgery), and any systemic or ocular pathology that could affect the cornea and tear film.
The central corneal thickness (CCT), axial length (AL), and keratometry measurements were performed with LenStar LS900 (Haag-Streit AG, Koeniz, Switzerland) because of its non-contact feature that facilitates paediatric examination and its very high-resolution ocular biometry measurements $[9,10]$. All the measurements were performed by one examiner $(\mathrm{GC})$.

All subjects underwent IOP measurements with a noncontact tonometer (NCT) and a Corvis ST tonometer in a random order with an interval of more than $1 \mathrm{~h}$. Three readings were taken with each instrument, with a 5-min pause between measurements taken with the same tonometer. The mean of the three readings was used for comparison and agreement. All IOP measurements were taken by the same examiner (CL) who was masked to the results analysis.

The ptotic eye was first examined twice, the fellow eye then underwent a Corvis ST scan three times, and the ptotic eye examination was repeated two or three times. The children were asked to adjust their chins and foreheads to an appropriate height and then to focus on the red-light emitting diode. The examination pattern was programmed for automatic release when alignment was achieved with the first Purkinje reflex of the cornea. The Corvis ST was set to take more than 4300 frames per second of the central $8 \mathrm{~mm}$ of the cornea with a high-speed Scheimpflug camera. The entire response of cornea deformation to an air puff was recorded and displayed as a video. A total of 140 digital frames were obtained and measured. The cornea experienced four distinct statuses: the first applanation (A1), the highest concavity (HC), the second applanation (A2), and the natural status (Table 1). Corvis_ST_1.2r1126 analysis software was used in this study. The corneal biomechanical property parameters were automatically measured immediately after the examination. Only the results given an "OK" in quality specification (QS) by the software were accepted and included in the final analysis. Inadequate quality Corvis ST images were excluded (Fig. 1).
Table 1 Corvis ST corneal deformation parameters

\begin{tabular}{ll}
\hline Length A1 & Cord length of the first-degree applanation \\
Velocity A1 & Corneal speed during the first-degree applanation \\
Time A1 & Time from starting until the first-degree applanation is reached \\
Length A2 & Cord length of the second-degree applanation \\
Velocity A2 & Corneal speed during the second-degree applanation \\
Time A2 & Time from starting until the second-degree applanation is reached \\
Time HC & Time from starting until the highest concavity is reached \\
Def Ampl HC & Maximum amplitude at the apex of highest concavity \\
Peak Dist HC & Distance of the two knees at the highest concavity \\
Radius HC & Central concave curvature at the highest concavity \\
IOP with Corvis & Intraocular pressure measured by Corvis \\
IOP corrected by Corvis & Intraocular pressure measured by Corvis with corrected \\
\hline
\end{tabular}




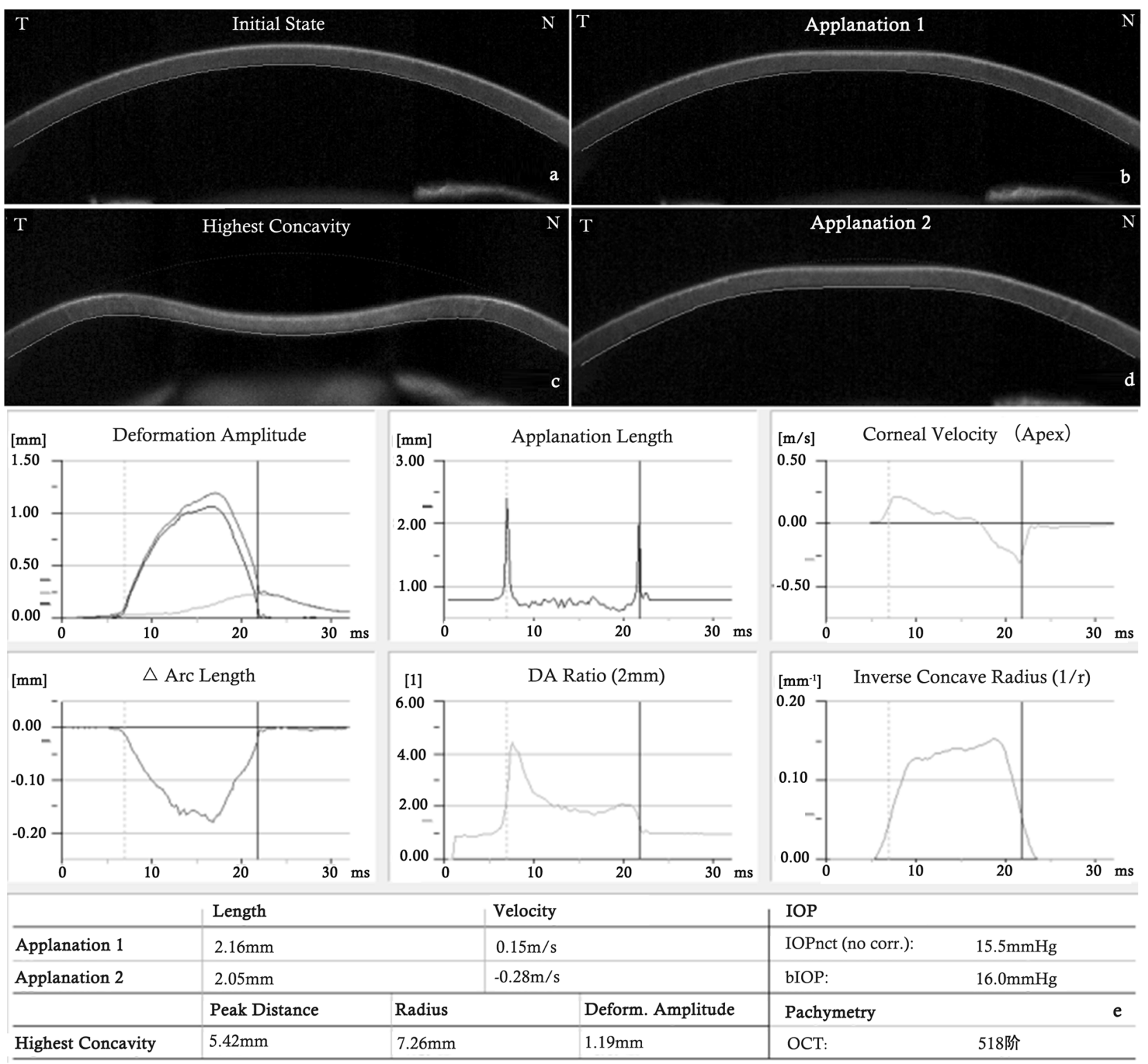

Fig. 1 Diagram of corneal response parameters on the images from the corneal visualization Scheimpflug technology. The top four figures are the four statuses: the initial status (a), the applanation 1 (A1) (b), the highest concavity (HC) (c), and the applanation 2 (A2) (d). The underlying diagram is the automatically measured corneal biomechanical property parameters $(\mathbf{e})$

\section{Statistical analysis of the data}

the clinical criteria, the choice of surgery was made according to LF. The levator resection was performed when LF was more than $5 \mathrm{~mm}$, and the frontalis suspension was chosen when $\mathrm{LF} \leq 5 \mathrm{~mm}$; additionally, the follow-up was performed according to a regular schedule, once per week in the first month and once per month after the second month. The same ophthalmic examinations were carried out 6 months after surgery using the same procedures. Patients lost to follow-up were excluded from the final analysis. Good functional and cosmetic outcomes were defined as a difference in MRD 1 less than $2 \mathrm{~mm}$ between the ptotic and fellow eyes and the absence of blepharoptosis or trichiasis.
The minimum required sample size for the study was calculated based on the previous study of changes in corneal biomechanical properties after long-term topical prostaglandin therapy by Corvis ST. Considering the previous study results for CCT and applanation time 1,20 pairs of eyes would be needed to detect a 5\% change in CCT with the power of a $95 \%$ confidence level [11].

Statistical analyses were performed using SPSS software version 17.0 (SPSS, Inc., Chicago, IL, USA). The means and standard deviations were calculated for the measured parameters. Snellen VA was converted to a $\log$ MAR value. 
Table 2 Demographic characteristics, corneal biomechanical parameters and comparison between ptotic and fellow eyes of congenital ptotic patients

\begin{tabular}{|c|c|c|c|}
\hline \multicolumn{4}{|l|}{ Characteristic (mean $\pm \mathrm{SD})$} \\
\hline Mean age, y & \multicolumn{3}{|l|}{$9.7 \pm 3.9$} \\
\hline Sex, male/female & \multicolumn{3}{|l|}{$19 / 10$} \\
\hline $\begin{array}{l}\text { Laterality of affected } \\
\text { eye, right/left }\end{array}$ & \multicolumn{3}{|l|}{$14 / 15$} \\
\hline \multicolumn{4}{|l|}{ Characteristics } \\
\hline $\mathrm{AL}(\mathrm{mm})$ & $23.09 \pm 1.06$ & $23.09 \pm 0.88$ & 0.944 \\
\hline IOP with NCT (mmHg) & $15.0 \pm 3.0$ & $14.5 \pm 2.8$ & 0.267 \\
\hline BCVA (logMAR) & $0.13 \pm 0.24$ & $0.01 \pm 0.08$ & $0.005^{* *}$ \\
\hline $\mathrm{SE}(\mathrm{D})$ & $0.38 \pm 2.21$ & $0.32 \pm 1.44$ & 0.826 \\
\hline $\mathrm{HPF}(\mathrm{mm})$ & $5.1 \pm 1.3$ & $9.0 \pm 1.2$ & $<0.001 * *$ \\
\hline MRD 1 (mm) & $-0.4 \pm 1.3$ & $3.5 \pm 1.2$ & $<0.001 * *$ \\
\hline $\mathrm{LF}(\mathrm{mm})$ & $4.1 \pm 1.8$ & $9.8 \pm 1.6$ & $<0.001 * *$ \\
\hline Mean SimK (D) & $42.72 \pm 1.79$ & $43.22 \pm 1.71$ & $0.014 *$ \\
\hline \multicolumn{4}{|l|}{$\begin{array}{l}\text { Corneal biomechanical } \\
\text { parameters }\end{array}$} \\
\hline Length A1 (mm) & $1.99 \pm 0.28$ & $1.98 \pm 0.32$ & 0.788 \\
\hline Velocity A1 (m/s) & $0.15 \pm 0.02$ & $0.16 \pm 0.01$ & $0.018 *$ \\
\hline Time A1 (ms) & $7.28 \pm 0.42$ & $7.17 \pm 0.32$ & $0.031 *$ \\
\hline Length A2 (mm) & $1.73 \pm 0.36$ & $1.72 \pm 0.28$ & 0.988 \\
\hline Velocity A2 (m/s) & $-0.32 \pm 0.08$ & $-0.34 \pm 0.08$ & 0.234 \\
\hline Time A2 (ms) & $21.84 \pm 0.44$ & $21.73 \pm 0.79$ & 0.474 \\
\hline Time HC (ms) & $16.84 \pm 0.42$ & $16.81 \pm 0.35$ & 0.768 \\
\hline Def Ampl HC (mm) & $1.08 \pm 0.12$ & $1.11 \pm 0.11$ & $0.028^{*}$ \\
\hline Peak Dist HC (mm) & $4.66 \pm 0.72$ & $4.89 \pm 0.34$ & 0.08 \\
\hline Radius HC (mm) & $6.51 \pm 0.87$ & $6.44 \pm 1.08$ & 0.74 \\
\hline $\mathrm{CCT}(\mu \mathrm{m})$ & $564 \pm 42$ & $551 \pm 40$ & $<0.001$ ** \\
\hline $\begin{array}{l}\text { IOP with } \\
\text { Corvis }(\mathrm{mmHg})\end{array}$ & $14.7 \pm 3.0$ & $13.8 \pm 2.3$ & $0.043 *$ \\
\hline $\begin{array}{l}\text { IOP with } \\
\text { corrected }(\mathrm{mmHg})\end{array}$ & $14.2 \pm 2.5$ & $13.8 \pm 1.4$ & 0.341 \\
\hline
\end{tabular}

* Paired t-test; $P<0.05$

$* * p<0.01$

Paired $t$ tests were used to detect the differences between ptotic eyes and fellow eyes in the same patient. Two-way analysis of variance was used to detect the changes in the corneal biomechanical parameters between ptotic eyes before and after surgery. Pearson correlation analysis was used to evaluate the relationships between age, AL, IOP, $\mathrm{CCT}$, spherical equivalent (SE), and Corvis ST parameters. A $p$ value of $<0.05$ was considered significant.

\section{Results}

A total of 31 children with unilateral congenital blepharoptosis were enroled in this study from July to August 2017. Two children were lost to follow-up during the 6-month follow-up. Among the remaining 29 patients, all ptotic eyes ( 29 eyes) and fellow eyes ( 29 eyes) were included in the final analyses. The mean age of the patients was $9.7 \pm 3.9$ years (range from 5 to 17 years old). Table 2 summarizes the demographic and clinical data of the subjects. The types of surgery that were performed included levator resection $(20.7 \%, 6 / 29)$ and frontalis suspension $(79.3 \%, 23 / 29)$. Good functional and cosmetic outcomes were achieved in $29(100 \%)$ patients, with satisfying symmetry during the follow-up.

The parameters of SE, AL, and IOP with NCT were not significant between ptotic and fellow eyes $(p>0.05$; Table 2). The BCVA, mean keratometry value, HPF, MRD 1 , and LF were lower in ptotic eyes $(p<0.05)$.

Ptotic eyes were considered to have less deformable corneas for several reasons (Table 2): (1) they reached the first applanation more slowly (with a longer Time A1 and smaller Velocity A1) $(p<0.05)$; (2) they showed a smaller concavity with a smaller DA HC $(p<0.05)$, smaller Peak Dist HC, and greater Radius HC; (3) they seemed more similar to a compressed cornea (with smaller Mean SimK); (4) and they showed a greater CCT.

Table 3 shows the results of correlation analyses. CCT was significantly positively correlated with Length A1 and IOP with Corvis and negatively correlated with IOP corrected by Corvis in ptotic eyes. The same tendency was noted in fellow eyes. Age was positively correlated with Radius $\mathrm{HC}$ and negatively correlated with Velocity A1, Velocity A2, Peak Dist $\mathrm{HC}$ in ptotic eyes. AL was positively correlated with Time A1 and negatively correlated with Velocity A1 and Time HC. However, the relationship of age and AL with Corvis ST parameters was less clear in fellow eyes.

Analysis of the study parameters after surgery revealed no significant differences in parameters between ptotic eyes and fellow eyes before and after surgery (Table 4).

\section{Discussion}

To the best of our knowledge, this study is the first to investigate the differences in corneal biomechanical properties between ptotic eyes and fellow eyes measured in unilateral congenital blepharoptosis patients using the novel Corvis ST NCT. After adjusting for factors that potentially influence corneal dynamic parameters, our results showed that significant differences in corneal biomechanical properties still exist between ptotic eyes and fellow eyes in patients with unilateral congenital blepharoptosis. This finding suggests that eyelid ptosis may have an obvious effect on corneal biomechanical properties.

Most previous studies on corneal biomechanical properties used the ocular response analyzer (ORA) as the measurement technology $[12,13]$. The ORA is the first 
Table 3 Correlations between corneal biomechanical parameters and demographic or ocular parameters

\begin{tabular}{|c|c|c|c|c|c|c|c|c|c|c|c|c|}
\hline & \multicolumn{2}{|l|}{ Age } & \multicolumn{2}{|l|}{ CCT } & \multicolumn{2}{|c|}{ Mean SimK } & \multicolumn{2}{|l|}{ SE } & \multicolumn{2}{|l|}{$\mathrm{AL}$} & \multicolumn{2}{|l|}{ IOP } \\
\hline & B & $p$ & B & $p$ & B & $p$ & B & $p$ & B & $p$ & B & $p$ \\
\hline \multicolumn{13}{|l|}{ Ptotic eyes } \\
\hline Length A1 & 0.025 & 0.876 & 25.434 & $0.003 * *$ & -1.05 & 0.808 & 0.185 & 0.33 & 0.167 & 0.362 & -6.803 & $0.003 * *$ \\
\hline Velocity A1 & -0.11 & 0.511 & -0.118 & 0.246 & 77.282 & 0.358 & -0.056 & 0.829 & -0.273 & 0.269 & 0.196 & 0.519 \\
\hline Time A1 & 0.13 & 0.439 & -0.042 & 0.714 & -4.231 & 0.509 & -2.233 & $0.016^{*}$ & 1.393 & $0.005 * *$ & -5.919 & $0.023^{*}$ \\
\hline Length A2 & 0.113 & 0.485 & 0.067 & 0.25 & 0.981 & 0.784 & 0.119 & 0.521 & -0.198 & 0.262 & -0.052 & 0.767 \\
\hline Velocity A2 & -26.089 & $<0.001 * *$ & 0.056 & 0.379 & 3.255 & 0.847 & 0.288 & 0.112 & -0.113 & 0.528 & 0.145 & 0.46 \\
\hline Time A2 & -0.07 & 0.628 & -0.06 & 0.308 & 0.601 & 0.722 & -0.109 & 0.638 & -0.108 & 0.627 & -0.168 & 0.388 \\
\hline Time HC & -0.117 & 0.439 & -0.025 & 0.677 & -1.482 & 0.496 & 0.002 & 0.994 & -0.239 & 0.207 & 0.011 & 0.953 \\
\hline Def Ampl HC & 0.177 & 0.406 & -0.078 & 0.304 & -7.227 & 0.554 & -0.335 & 0.133 & 0.203 & 0.349 & 0.004 & 0.988 \\
\hline Peak Dist HC & -3.193 & $0.001 * *$ & 0.031 & 0.103 & -1.592 & 0.585 & 0.217 & 0.26 & 0.019 & 0.92 & -0.156 & 0.331 \\
\hline Radius HC & 1.5 & $0.024 *$ & -0.029 & 0.651 & 2.237 & 0.224 & 0.075 & 0.756 & -0.263 & 0.25 & 0.138 & 0.499 \\
\hline $\begin{array}{l}\text { IOP with } \\
\text { Corvis (mmHg) }\end{array}$ & 0.11 & 0.516 & 23.56 & $<0.001 * *$ & -0.681 & 0.639 & 0.507 & 0.113 & -0.319 & 0.307 & 1.3 & $0.001 * *$ \\
\hline \multirow{2}{*}{$\begin{array}{l}\text { IOP with } \\
\text { corrected }(\mathrm{mmHg})\end{array}$} & 0.057 & 0.713 & -22.644 & $<0.001 * *$ & 1.169 & 0.391 & 0.389 & 0.108 & -0.15 & 0.529 & -0.31 & 0.231 \\
\hline & \multicolumn{2}{|c|}{$R^{2}=0.613$} & \multicolumn{2}{|c|}{$R^{2}=0.941$} & \multicolumn{2}{|c|}{$R^{2}=0.095$} & \multicolumn{2}{|c|}{$R^{2}=0.185$} & \multicolumn{2}{|c|}{$R^{2}=0.25$} & \multicolumn{2}{|c|}{$R^{2}=0.441$} \\
\hline \multicolumn{13}{|l|}{ Fellow eyes } \\
\hline Length A1 & -0.03 & 0.971 & 0.038 & 0.831 & -0.064 & 0.999 & 0.218 & 0.971 & -0.158 & 0.413 & -1.678 & 0.639 \\
\hline Velocity A1 & -0.003 & 0.954 & -0.075 & 0.78 & 0.137 & 0.921 & -0.109 & 0.954 & -0.154 & 0.451 & 11.421 & 0.88 \\
\hline Time A1 & 6.605 & $0.006 * *$ & -0.063 & 0.471 & 0.077 & 0.836 & -2.906 & $0.001 * *$ & 0.234 & 0.253 & -4.046 & 0.386 \\
\hline Length A2 & -0.219 & 0.989 & 0.035 & 0.931 & 0.412 & 0.917 & -0.034 & 0.989 & -0.192 & 0.342 & 2.596 & 0.422 \\
\hline Velocity A2 & -0.338 & 0.972 & 0.093 & 0.943 & -0.083 & 0.855 & 0.167 & 0.972 & -0.127 & 0.521 & -15.972 & 0.186 \\
\hline Time A2 & -0.118 & 0.793 & 0.046 & 0.674 & -0.016 & 0.922 & -0.261 & 0.793 & 0.177 & 0.405 & -0.679 & 0.645 \\
\hline Time HC & -0.02 & 0.871 & -0.068 & 0.133 & 2.181 & $0.13 *$ & -0.065 & 0.871 & -1.031 & $0.05^{*}$ & -1.521 & 0.572 \\
\hline Def Ampl HC & 0.137 & 0.645 & -0.104 & 0.374 & 0.033 & 0.842 & -0.154 & 0.645 & 0.043 & 0.844 & -1.149 & 0.939 \\
\hline Peak Dist HC & -0.07 & 0.939 & -0.106 & 0.899 & -0.125 & 0.895 & -0.131 & 0.939 & 0.135 & 0.506 & 0.584 & 0.784 \\
\hline Radius HC & -0.069 & 0.945 & 0.011 & 0.83 & 0.01 & 0.974 & -0.003 & 0.945 & 0.048 & 0.811 & -0.628 & 0.409 \\
\hline $\begin{array}{l}\text { IOP with } \\
\text { Corvis }(\mathrm{mmHg})\end{array}$ & -0.121 & 0.473 & 21.744 & $<0.00 * *$ & 0.04 & 0.991 & 0.387 & 0.473 & -0.033 & 0.869 & 0.935 & 0.219 \\
\hline \multirow{2}{*}{$\begin{array}{l}\text { IOP with } \\
\text { corrected }(\mathrm{mmHg})\end{array}$} & -0.236 & 0.759 & -18.643 & $<0.00 * *$ & 0.133 & 0.998 & 0.301 & 0.759 & -0.022 & 0.908 & -0.099 & 0.882 \\
\hline & \multicolumn{2}{|c|}{$R^{2}=0.248$} & \multicolumn{2}{|c|}{$R^{2}=0.888$} & \multicolumn{2}{|c|}{$R^{2}=0.324$} & \multicolumn{2}{|c|}{$R^{2}=0.358$} & \multicolumn{2}{|c|}{$R^{2}=0.116$} & \multicolumn{2}{|c|}{$R^{2}=-0.061$} \\
\hline
\end{tabular}

* Pearson correlation analysis; $P<0.05$

$* * p<0.01$

commercially available technology for the measurement of corneal biomechanical properties. The Corvis ST provides improvements in this technology, and newer instruments may provide even better in vivo characterizations of the complex biomechanical characteristics of the cornea [14]. The relationship between Corvis ST tonometry and ORA measurements has been studied. Most of the Corvis ST parameters showed significant relationships with the ORA parameters [15].

In the present study, the SE and AL of ptotic and fellow eyes were not significantly different before surgery. This finding was contrary to the hypothesis that visual deprivation during infancy may deregulate axial growth of the eye.
However, this less predictable finding was also reported by other studies [16]. These studies found that patients with unilateral dense congenital cataracts and unilateral complete blepharoptosis did not show consistent elongation of the involved eye. This finding meant that unlike in monkeys where axial elongation usually prevails, this effect of visual deprivation is less predictable in humans.

Human corneal tissue is considered a viscoelastic material with measurable properties. The corneal stroma is composed of type 1 collagen and an extracellular matrix mainly represented by proteoglycans and glycosaminoglycans, which are secreted by corneal stromal cells [17]. Previous studies found that a symmetrical arrangement of 
Table 4 Comparison of the pre-surgery and post-surgery difference between ptotic and fellow eyes

\begin{tabular}{lccl}
\hline Characteristic & Pre-surgery & Post-surgery & $P$ value \\
\hline Length A1 & $1.99 \pm 0.28$ & $1.99 \pm 0.28$ & 0.986 \\
Velocity A1 & $0.15 \pm 0.02$ & $0.15 \pm 0.01$ & 0.975 \\
Time A1 & $7.28 \pm 0.42$ & $7.31 \pm 0.33$ & 0.853 \\
Length A2 & $1.72 \pm 0.36$ & $1.72 \pm 0.32$ & 0.913 \\
Velocity A2 & $-0.32 \pm 0.08$ & $-0.34 \pm 0.06$ & 0.349 \\
Time A2 & $21.84 \pm 0.44$ & $21.84 \pm 0.35$ & 0.991 \\
Time HC & $16.84 \pm 0.42$ & $16.87 \pm 0.36$ & 0.764 \\
Def Ampl HC & $4.66 \pm 0.72$ & $4.69 \pm 0.47$ & 0.456 \\
Peak Dist HC & $6.51 \pm 0.87$ & $6.57 \pm 0.53$ & 0.798 \\
Radius HC & $1.08 \pm 0.12$ & $1.10 \pm 0.06$ & 0.95 \\
CCT & $564 \pm 42$ & $565 \pm 33$ & 0.897 \\
IOP with Corvis $(\mathrm{mmHg})$ & $14.7 \pm 3.0$ & $15.4 \pm 2.2$ & 0.06 \\
IOP with corrected $(\mathrm{mmHg})$ & $14.2 \pm 2.5$ & $14.1 \pm 2.0$ & 0.924 \\
\hline
\end{tabular}

* Two-way analysis of variance; $P<0.05$

$* * p<0.01$

corneal collagen leads to symmetrical corneal biomechanical parameters in both eyes of healthy people [18, 19]. The biomechanical properties of the cornea are modified by metabolic and lifetime environmental factors, especially UV exposure, so the $\mathrm{CH}$ and $\mathrm{CRF}$ mean values are significantly lower in subjects exposed to higher UV radiation than in a reference group [20]. Chronic corneal disturbances induced by UVB rays may include apoptosis of corneal cells, inhibition of cell proliferation, and cell autolysis of central layers of the epithelium, leading to thinning of the corneal epithelium [21]. Daxer et al. [22] demonstrated an increase in collagen fibril diameter and intermolecular Bragg spacing with increasing age [22]. These changes in corneal ultrastructure altered the viscoelastic deformation properties and biomechanical behaviour [19]. We speculate that the ptotic eyelid pressure in our patients induced abnormal development of the cornea and that persistent covering would reduce the UV exposure of the ptotic eyes, which might affect corneal restructuring during development. Changes in the corneal biomechanical properties may reflect structural changes in the ground substance of the cornea.

According to the linear model of the ORA and Corvis ST parameters, we determined that the $\mathrm{CH}$ and $\mathrm{CRF}$ were greater in ptotic eyes than in fellow eyes in patients with congenital ptosis [15]. The slower Velocity A1, longer Time A1, longer Time A2, and lower Velocity A2 indicated that ptotic eyes had a less deformable cornea. The results were generally consistent with the deformation properties reported in previous studies of adult corneas [23-25].

In our study, we found a significantly thicker CCT in ptotic eyes than in fellow eyes. Savino et al. [26] reported no significant differences in CCT between preoperative and postoperative examinations. We have also followed up our patients for 6 months after surgery and found no significant changes in CCT, which suggested that the thickening of the central cornea was not temporary or the result of a deficiency in tear evaporation.

We found that CCT was significantly positively correlated with Length A1 and IOP determined with Corvis and negatively correlated with IOP corrected by Corvis in ptotic eyes. We speculated that CCT was the most pivotal factor that changed in ptotic eyes and subsequently affected the other parameters. However, the mechanism of corneal thickening remains uncertain. We plan further studies on tear analysis and corneal collagen fibrils.

The mean age of our study participants was $9.7 \pm 3.9$ years. A population-based study has shown that $\mathrm{CH}$ and CRF values continue to decrease with increasing age [20]. Whether the surgical timing for congenital ptosis affects the changes in preoperative and postoperative corneal biomechanical properties is unknown. To the best of our knowledge, there are no other studies about this subject. However, many studies have focused on refractive changes after ptosis surgery. Theoretically, lifting the ptotic eyelid should alleviate this mechanical force and correct the astigmatism. However, several studies have shown increased astigmatism following ptosis surgery in the paediatric population $[27,28]$. Cadera et al. found that the operative group showed an average increase in cylinder of $0.30 \mathrm{D}$ after surgery. Further analysis of age and astigmatism changes showed that the younger group ( $<4$ years old $)$ had an average decrease in cylinder of $0.20 \mathrm{D}$, while the older group was noted to have an average increase in cylinder of $0.50 \mathrm{D}$ [27]. In our study, we divided the patients by age into adolescents (14 patients at 9-18 years old) and children (15 patients at 5-9 years old). The changes in preoperative and postoperative corneal biomechanical properties were not significant in the two age groups (not present). However, it is important to note the small number of patients when separated into age groups. Larger sample size studies are needed.

One strength of this study is that it represents the first longitudinal research on the effects of congenital blepharoptosis on corneal biomechanical properties measured by Corvis ST tonometry and the first to follow-up the differences between ptotic and fellow eyes before and after surgery. However, several limitations in the present study should not be ignored. First, the sample size was limited; however, most parameters were sufficient to show a significant difference between ptotic eyes and fellow eyes, which is consistent with previous studies. Second, the research subjects were children under 17 years old, and their adaptability to the examinations was dubious. We endeavoured to reduce the uncertainty by including children older than 5 years who were able to cooperate with the 
examinations. Repeated examinations were performed, and only the results with an "OK" in QS by the software were included in the final analysis. Third, the follow-up after ptosis surgery was not sufficiently long. We found no significant changes 6 months after surgery, whereas we might see significant differences after a longer follow-up. Finally, the poor reliability and reproducibility of some Corvis ST parameters have been mentioned in other studies. We aimed to reduce the effects of diurnal variations by comparing the differences in values between ptotic and fellow eyes of the same patients before and after surgery.

In conclusion, the slower Velocity A1, longer Time A1, longer Time A2, and lower Velocity A2 indicated that ptotic eyes had a less deformable cornea. CCT had an important influence on corneal biomechanical parameters, while age, $\mathrm{SE}$, mean keratometry, and AL exerted little influence on the Corvis ST measurements in the children studied here. Further studies with longer follow-up times are needed to assess corneal biomechanical changes in children with congenital blepharoptosis after ptosis surgery.

\section{Summary}

\section{What was known before}

- Congenital blepharoptosis is frequently associated with amblyopia, refractive error, anisometropia, and strabismus.

- The ptotic eyes have more severe astigmatism and more oblique astigmatism than their fellow eyes.

- A significant change in astigmatism has been detected after surgery.

\section{What this study adds}

- Congenital blepharoptosis causes significant corneal biomechanical changes measured by Corvis ST.

- The ptotic eyes had thicker and less deformable corneas.

- We speculated that the CCT was the most pivotal factor that changed in ptotic eyes, and subsequently affected the other parameters.

Funding This work is supported by grants from the Special Fund Project of Science and Technology in Guangdong Province (2018YJ036), and the Research and Development and Upgrading Fund of Enterprises in Guangdong Province (2013B022200002).

\section{Compliance with ethical standards}

Conflict of interest The authors declare that they have no conflict of interest.
Publisher's note Springer Nature remains neutral with regard to jurisdictional claims in published maps and institutional affiliations.

\section{References}

1. SooHoo JR, Davies BW, Allard FD, Durairaj VD. Congenital ptosis. Surv Ophthalmol. 2014;59:483-92.

2. Srinagesh V, Simon JW, Meyer DR, Zobal-Ratner J. The association of refractive error, strabismus, and amblyopia with congenital ptosis. J AAPOS. 2011;15:541-4.

3. Oral Y, Ozgur OR, Akcay L, Ozbas M, Dogan OK. Congenital ptosis and amblyopia. J Pediatr Ophthalmol Strabismus. 2010;47:101-4.

4. Skaat A, Fabian D, Spierer A, Rosen N, Rosner M, Ben SG. Congenital ptosis repair-surgical, cosmetic, and functional outcome: a report of 162 cases. Can J Ophthalmol. 2013;48:93-98.

5. Zhu T, Ye X, Xu P, et al. Changes of corneal tomography in patients with congenital blepharoptosis. Sci Rep. 2017;7:6580.

6. Paik J, Kim S, Park SH, Yang S. Refractive error characteristics in patients with congenital blepharoptosis before and after ptosis repair surgery. BMC Ophthalmol. 2016;16:177-83.

7. Trivizki O, Shahar J, Levinger S, Levinger E. Air-pulse corneal applanation signal curve parameters for characterization of astigmatic corneas. Cornea. 2014;33:721-5.

8. Griepentrog GJ, Diehl NN, Mohney BG. Incidence and demographics of childhood ptosis. Ophthalmology. 2011; 118:1180-3.

9. Gursoy H, Sahin A, Basmak H, Ozer A, Yildirim N, Colak E. Lenstar versus ultrasound for ocular biometry in a pediatric population. Optom Vis Sci. 2011;88:912-9.

10. O’Donnell C, Hartwig A, Radhakrishnan H. Comparison of central corneal thickness and anterior chamber depth measured using LenStar LS900, Pentacam, and Visante AS-OCT. Cornea. 2012;31:983-8.

11. Wu N, Chen Y, Yu X, Li M, Wen W, Sun X. Changes in corneal biomechanical properties after long-term topical prostaglandin therapy. PLOS ONE. 2016;11:e155527.

12. Glass DH, Roberts CJ, Litsky AS, Weber PA. A viscoelastic biomechanical model of the cornea describing the effect of viscosity and elasticity on hysteresis. Investig Ophthalmol Vis Sci. 2008;49:3919-26.

13. Touboul D, Roberts C, Kerautret J, et al. Correlations between corneal hysteresis, intraocular pressure, and corneal central pachymetry. J Cataract Refract Surg. 2008;34:616-22.

14. Chua J, Nongpiur ME, Zhao W, et al. Comparison of corneal biomechanical properties between Indian and Chinese adults. Ophthalmology. 2017;124:1271-9.

15. Matsuura M, Hirasawa $\mathrm{K}$, Murata $\mathrm{H}$, et al. The relationship between Corvis ST tonometry and ocular response analyzer measurements in eyes with glaucoma. PLOS ONE. 2016;11: e161742.

16. von Noorden GK, Lewis RA. Ocular axial length in unilateral congenital cataracts and blepharoptosis. Investig Ophthalmol Vis Sci. 1987;28:750-2.

17. Robert L, Legeais JM, Robert AM, Renard G. Corneal collagens. Pathol Biol. 2001;49:353-63.

18. He M, Ding H, He H, Zhang C, Liu L, Zhong X. Corneal biomechanical properties in healthy children measured by corneal visualization scheimpflug technology. BMC Ophthalmol. 2017; 17:70-6.

19. Kotecha A. What biomechanical properties of the cornea are relevant for the clinician? Surv Ophthalmol. 2007;52 Suppl: S109-S114. 
20. Schweitzer C, Korobelnik JF, Boniol M, et al. Associations of biomechanical properties of the cornea with environmental and metabolic factors in an elderly population: the ALIENOR Study. Investig Ophthalmol Vis Sci. 2016;57:2003-11.

21. Ardan T, Nemcova L, Bohuslavova B, et al. Reduced levels of tissue inhibitors of metalloproteinases in UVB-irradiated corneal epithelium. Photochem Photobiol. 2016;92:720-7.

22. Daxer A, Misof K, Grabner B, Ettl A, Fratzl P. Collagen fibrils in the human corneal stroma: structure and aging. Investig Ophthalmol Vis Sci. 1998;39:644-8.

23. Salvetat ML, Zeppieri M, Tosoni C, Felletti M, Grasso L, Brusini P. Corneal deformation parameters provided by the corvis-ST pachy-tonometer in healthy subjects and glaucoma patients. J Glaucoma. 2015;24:568-74.
24. Lanza M, Cennamo M, Iaccarino S, et al. Evaluation of corneal deformation analyzed with Scheimpflug based device in healthy eyes and diseased ones. Biomed Res Int. 2014;2014:748671.

25. Valbon BF, Ambrosio RJ, Fontes BM, Luz A, Roberts CJ, Alves MR. Ocular biomechanical metrics by CorVis ST in healthy Brazilian patients. J Refract Surg. 2014;30:468-73.

26. Savino G, Battendieri R, Riso M, et al. Corneal topographic changes after eyelid ptosis surgery. Cornea. 2016;35:501-5.

27. Cadera W, Orton RB, Hakim O. Changes in astigmatism after surgery for congenital ptosis. J Pediatr Ophthalmol Strabismus. 1992;29:85-88.

28. Chisholm S, Costakos DM, Harris GJ. Surgical timing for congenital ptosis should not be determined solely by the presence of anisometropia. Ophthalmic Plast Reconstr Surg. 2019;35:374-7. 